\title{
Investigando a aplicação de ferramentas de Edge Computing na área de Indústria 4.0
}

\author{
Matheus L. da Silva and César A. F. De Rose \\ ${ }^{1}$ Escola Politécnica - Pontifícia Universidade Católica do Rio Grande do Sul (PUCRS) \\ Porto Alegre - RS - Brazil \\ matheus.lyraledu.pucrs.br, cesar.deroselpucrs.br
}

\begin{abstract}
Resumo. Na última década, a área de cloud computing transformou a indústria de TI com serviços sob-demanda. Recentemente, o surgimento de outras tecnologias como IoT e a indústria 4.0 vem ocasionando um aumento massivo na produção de dados. Neste estudo discutimos como a área de edge computing é vista como uma possível solução para esta nova problemática juntamente de projetos já apresentados pela indústria e comunidade acadêmica.
\end{abstract}

\section{Introdução}

A área de edge computing propõe que servidores estejam localizados geograficamente mais próximos do usuário final do que os servidores em nuvem. Dentro desse contexto, serviços como processamento e armazenamento, ocorrem em parte na borda da rede, o que permite a redução do consumo de largura de banda e latência na comunicação direta com os dispositivos de usuário. A literatura, de forma geral, coloca esta área em uma posição essencial para baratear o processamento de dados e para a continuidade na utilização da infraestrutura atual dos serviços de cloud computing.

Devido a natureza das áreas de IoT e indústria 4.0, aplicações destes segmentos tendem a ser sensíveis a atrasos. A proposta da proximidade geográfica dos dispositivos de usuário, bem como das máquinas industrias, tende a fornecer baixa latência na comunicação, o que é um fator importante dado o cenário considerado. Deste modo, embora edge nodes tenham um menor poder de processamento se comparado aos servidores em nuvem, ainda fornecem melhor qualidade de serviço (QoS) [Mao et al. 2017]. Além do crescimento explosivo na demanda por serviços com alta largura de banda devido aos milhões de dispositivos de usuários, a indústria de manufatura também evoluiu em suas formas de fazer produtos [Salman et al. 2015]. A Indústria 4.0, como é chamada, conecta a área de IoT com técnicas de manufatura para permitir que sistemas compartilhem, analisem e utilizem informações para orientar ações inteligentes durante o processo de fabricação. Espera-se que este uso de informações se intensifique ainda mais devido aos avanços esperados na tecnologia $5 \mathrm{G}$ para os próximos anos [Salman et al. 2015]. Como a área de edge computing pode potencializar as áreas de cloud computing, IoT e indústria 4.0, essa área vem se tornando cada vez mais um tópico de pesquisa atraente.

\section{Visão geral e trabalhos futuros}

De forma geral, os conceitos de edge computing se concentram em métricas importantes, como delay e high-bandwidth, latency cost e consumo de energia [Abbas et al. 2017]. Além da aplicação dentro de fábricas, outros cenários de aplicação emergentes são as 
áreas de healthcare, mobile applications, big data, video analytics e connected vehicles [Qadri et al. 2020, Abbas et al. 2017].

Além dos esforços da comunidade científica, existem projetos independentes e privados que investigam esta área. Um exemplo é o $\mathrm{FOGFLOW}^{1}$, um projeto focado na área de edge computing oriundo de outra iniciativa chamada FIWARE ${ }^{2}$. Outro exemplo é o $\mathrm{I}_{4} \mathrm{MS}^{3}$, um projeto financiado pela Comissão Europeia com foco na inovação tecnológica considerando a $4^{a}$ revolução industrial. Além disso, empresas focadas em infraestrutura de nuvem, vem se adaptando e começam a oferecer novos serviços localizados na borda da rede. Alguns exemplos são o Amazon CloudFront ${ }^{4}$, Microsoft Azure Edge Zones ${ }^{5}$ e IBM Edge Computing ${ }^{6}$. Entre as soluções, vemos o uso de Kubernetes nos serviços de edge da IBM, o que parece ser uma opção interessante para aplicativos escaláveis. $\mathrm{O}$ framework FOGFLOW também menciona o uso interno da engine do docker, permitindo a alocação de diversas tarefas em workers que estão associados a recursos computacionais na nuvem ou apenas em um nó na extremidade da rede[Cheng et al. 2017].

A comunidade acadêmica vem pesquisando novas soluções no que diz respeito ao uso dos recursos computacionais dentro da proposta de edge computing e a indústria também vem demonstrando interesse neste tópico. Deste modo, pode-se notar um aumento significativo de iniciativas e parcerias diversas destes segmentos para investigar como as áreas de IoT e edge computing podem oferecer suporte a empresas no setor 4.0. Acreditamos que a área de edge computing ocupará um importante papel nos próximos anos, buscando avanços para o processamento de dados na borda da rede.

\section{Referências}

Abbas, N., Zhang, Y., Taherkordi, A., and Skeie, T. (2017). Mobile edge computing: A survey. IEEE Internet of Things Journal, 5(1):450-465.

Cheng, B., Solmaz, G., Cirillo, F., Kovacs, E., Terasawa, K., and Kitazawa, A. (2017). Fogflow: Easy programming of iot services over cloud and edges for smart cities. IEEE Internet of Things Journal, 5(2):696-707.

Mao, Y., You, C., Zhang, J., Huang, K., and Letaief, K. B. (2017). A survey on mobile edge computing: The communication perspective. IEEE Communications Surveys \& Tutorials, 19(4):2322-2358.

Qadri, Y. A., Nauman, A., Zikria, Y. B., Vasilakos, A. V., and Kim, S. W. (2020). The future of healthcare internet of things: A survey of emerging technologies. IEEE Communications Surveys Tutorials, pages 1-1.

Salman, O., Elhajj, I., Kayssi, A., and Chehab, A. (2015). Edge computing enabling the internet of things. In 2015 IEEE 2nd World Forum on Internet of Things (WF-IoT), pages 603-608.

\footnotetext{
${ }^{1}$ https://fogflow.readthedocs.io/en/latest/

${ }^{2}$ https://fiware-device-simulator.readthedocs.io/en/latest/

${ }^{3}$ https://i4ms.eu/

${ }^{4}$ https://aws.amazon.com/cloudfront/

${ }^{5}$ https://azure.microsoft.com/solutions/low-latency-edge-computing/

${ }^{6}$ https://www.ibm.com/partnerworld/cloud/edge-computing
} 